\title{
Least action criteria for blind separation of structural modes
}

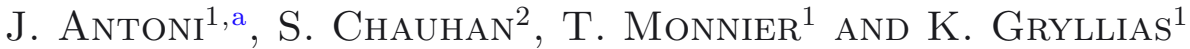 \\ 1 Laboratoire Vibrations Acoustique, University of Lyon, 69621 Villeurbanne, France \\ 2 Bruel \& Kjaer Sound and Vibration Measurement A/S, Skodsborgvej 307, 2850 Naerum, Denmark
}

Received 9 August 2013, Accepted 20 December 2013

\begin{abstract}
It was recently shown that blind source separation (BSS), as originally developed in the signal processing community, can be used in operational modal analysis to separate the responses of a structure into its individual modal contributions. This, in turn, allows the application of simple single-of-degreefreedom techniques to identify the modal parameters of interest. Several publications have recently attempted to give a posteriori physical interpretations to BSS - as initially developed in telecommunication signal processing - when applied to the field of structural dynamics. This paper proposes to follow the route the other way round. It shows that several separation criteria purposely dedicated to operational modal analysis can be deduced from general physical considerations. Three such examples are introduced, based on very different properties that uniquely characterise a structural mode. The first criterion, coined the "principle of shortest envelope", conjectures that the envelope of a modal response has, among all possible envelopes, the shortest length. That such a principle leads to the governing differential equation of a single-degree-of-freedom oscillator is proved from calculus of variation. The second criterion, coined the "principle of minimum spectral variance", conjectures that the frequency spectrum of a structural mode is maximally concentrated around its central frequency. Finally, the third criterion, coined the "principle of least spectral complexity", states that a structural mode has the lowest possible entropy in the frequency domain. All three criteria can be expressed in terms of a mixing matrix whose columns contain the unknown mode shapes. The recovery of the latter is then trivially achieved by minimising the criteria. Extensive simulations show that the proposed criteria lead to figures of merit very similar to those of the state-of-the-art, while at the same time providing physical insight that other algorithms issued form the signal processing community may dramatically lack.
\end{abstract}

Key words: Operational modal analysis / blind source separation / least action principle / calculus of variation / structural dynamics

Résumé - Principes de moindre action pour la séparation aveugle de modes. Des recherches récentes ont montré que certaines méthodes de séparation aveugle de sources, initialement développées pour les signaux de télécommunication, permettent de décomposer les réponses vibratoires d'une structure en ses différentes contributions modales. L'avantage qui s'ensuit pour l'analyse modale opérationnelle est une identification immédiate des paramètres modaux par application des techniques traditionnelles dédiées aux systèmes à un degré de liberté, et ceci sans connaissance des forces excitatrices appliquées à la structure. L'approche est dite «aveugle», car elle ne nécessite aucune connaissance préalable des paramètres structuraux. Plusieurs publications ont récemment tenté de donner a posteriori une interprétation physique aux méthodes de séparation aveugle de sources, lorsque appliquées en dynamique des structures. Cette communication propose de suivre la démarche inverse en montrant que des critères de séparation inédits et ad hoc peuvent être définis suivant des considérations physiques générales. Trois exemples sont donnés. Le premier critère, dit de «moindre longueur de l'enveloppe », stipule que l'enveloppe d'une réponse modale est la plus courte parmi toutes les trajectoires possibles. L'équation du mouvement qui en découle est obtenue par calcul variationel. Le second critère, dit de « moindre variance spectrale », stipule que la densité spectrale d'une réponse modale est la plus concentrée possible autour d'une fréquence centrale. Enfin, le troisième critère dit de «moindre complexité spectrale » stipule qu'une réponse modale a la plus faible entropie possible dans le domaine fréquentiel. Tous ces critères peuvent être exprimés selon une intégrale de moindre action en fonction de la matrice de mélange qui contient

${ }^{a}$ Corresponding author: jerome.antoni@insa-lyon.fr 
les déformées modales inconnues. L'estimation de celles-ci est solution de leur minimisation. Plusieurs simulations montrent que l'approche proposée donne lieu à des résultats de séparation très similaires à ceux de l'état de l'art, l'avantage principal étant d'être issus de considérations physiques spécifiques à la problématique de l'analyse modale opérationnelle contrairement aux autres méthodes de séparation aveugle de sources.

Mots clés : Analyse modale opérationnelle / séparation aveugle de sources / principe de moindre action / calcul des variations / dynamique des structures

\section{Nomenclature}

\begin{tabular}{|ll|}
\hline BSS & Blind Source Separation \\
MAC & Modal Acceptance Criterion \\
MSEnt & Minimum Spectral Entropy \\
MSV & Minimum Spectral Variance \\
OMA & Operational Modal Analysis \\
SHE & Shortest Envelop \\
SNR & Signal to Noise Ratio \\
SOBI & Second Order Blind Identification \\
\hline
\end{tabular}

\section{Introduction}

Operational modal analysis (OMA) aims at identifying the modal properties of a structure given only its responses in ambient environment, that is without having access to actual excitation forces. This happens whenever specific excitation setups cannot be installed, because of technological or financial reasons, or simply because artificial excitations would not be representative enough of the actual in-situ force field. Many techniques have been proposed over the last decades to solve the OMA problem from different angles of attack - see e.g. [1,2]. One recent and elegant solution consists of viewing the modal expansion of a set of structural responses as a mixture of modal coordinates - the "source signals" - where the mode shapes fill the columns of a mixing matrix. Then, under quite mild assumptions concerning only the mutual independence of the sources, it is possible to identify all constituents of the mixture up to an arbitrary scaling of the mode shapes [3-20]. In turn, standard single-degreeof-freedom techniques can be applied on the separated modal coordinates to identify the global modal parameters. Such an approach is referred to as "blind", since it does not require the knowledge of the mass and stiffness matrices, as is usually the case to recover mode shapes - e.g. from an eigenvalue decomposition. It is also different from classical modal identification, since it is not based on any parametric model. The current state-ofthe-art in the context of operational modal analysis is rooted on the so-called Second-Order Blind Identification (SOBI) algorithm, which has proven extremely robust: its principle consists in separating sources - i.e. modal coordinates - which are least mutually correlated at several time-lags $[4,6-9,20]$. This was shown to work surprisingly well, even though the assumption of mutual decorrelation of modes is not truly fulfilled as soon as the system is non-conservative (i.e. presence of damping). Like many other BSS algorithms, SOBI was initially discovered in the signal processing community [21-25] and the fact that it found pertinent applications in the field of structural dynamics might be more a fortunate coincidence than the result of an intended research program. As a matter of fact, several publications have recently attempted to give a posteriori physical interpretation to $\operatorname{SOBI}[3,4,9,20]$.

The objective of this paper is specifically to tackle the issue the other way round. Given the mixture model suggested by the modal expansion theorem, can one devise ad hoc criteria - inspired from general physical principles - that can separate individual modes?

The answer to this question is shown to be positive. In addition, it appears quite surprisingly that many such criteria exist, which result in as many new separation algorithms. This paper explores three of them, based on very different properties that uniquely characterise a structural mode. The first criterion, coined the "principle of shortest envelope", conjectures that the envelope of a modal response has, among all possible envelopes, the shortest length. That such a principle leads to the governing differential equation of a single-degree-of-freedom oscillator is proved from calculus of variation. The second criterion, coined the "principle of minimum spectral variance", conjectures that the frequency spectrum of a structural mode is maximally concentrated around its central frequency. Finally, the third criterion, coined the "principle of least spectral complexity", states that a structural mode has the lowest possible entropy in the frequency domain. All three criteria can be expressed in terms of a mixing matrix whose columns contain the unknown mode shapes. The recovery of the latter is then trivially achieved by minimising the criteria. One difference with SOBI - and other related BSS algorithms - is that mode shapes are recovered one by one, in a deflation way, rather than simultaneously in batch way. Extensive simulations show that the proposed criteria lead to figures of merit very similar to those of SOBI. Their main advantage is yet conceptual rather than algorithmic; indeed, least action principles provide physical insight into the mechanism of BSS that other algorithms issued form the signal processing community may dramatically lack. No doubt that other physical properties could be exploited to define still new separation criteria by following the lines of this paper.

\section{Blind separation of modal responses}

It was recently shown that blind source separation (BSS), as originally developed in the signal processing community, can be used in operational modal analysis 
(OMA) to separate the responses of a structure into its individual modal contributions. The central idea is to view the modal expansion of a set of responses as a mixture of modal coordinates. Namely, let $y_{i}(t), i=1, \ldots, m$, be the structural responses, measured by a set of $m$ sensors, of an $n$-degree-of-freedom system with modal coordinate $\eta_{j}(t), j=1, \ldots, n$, and mode shape components $\phi_{i j}(t)$. According to the modal expansion theorem,

$$
y_{i}(t)=\sum_{j=1}^{n} \phi_{i j} \eta_{j}(t)+n_{i}(t)
$$

where the extra term $n_{i}(t)$ accounts for possible measurement noise. Put into a matrix form, this reads

$$
\mathbf{y}(t)=\boldsymbol{\Phi} \boldsymbol{\eta}(t)+\mathbf{n}(t)
$$

where $\mathbf{x}(t)=\left[x_{1}(t) \ldots x_{n}(t)\right]^{T}(\mathbf{x}=\mathbf{y}, \boldsymbol{\eta}$ and $\mathbf{n})$. Equation (2) exhibits a strong similarity with the mixture model found in BSS, where the modal coordinates play the roles of the sources and the mode shapes fill the columns of the mixing matrix. This has suggested the application of BSS on the measured structural responses $y_{i}(t)$ in an attempt to recover the modal matrix $\boldsymbol{\Phi}$ together with the individual modal coordinates $\eta_{j}(t)$ with no other assumption than the mutual independence of the latter. One prominent tool in the state-of-the-art is the SOBI algorithm, which achieves separation of sources by forcing their decorrelation at several time-lags. Specifically, let $\mathbf{R}_{y}(\tau)=\left\langle\mathbf{y}(t) \mathbf{y}(t-\tau)^{H}\right\rangle$ be the correlation matrix of the measurements under steady state regime at time-lag $\tau$, where $\langle\ldots\rangle=\lim _{T \rightarrow \infty} T^{-1} \int_{T} \ldots \mathrm{d} t$ denotes the time-averaging operator and ${ }^{H}$ stands for the complex conjugate operation (complex data will be considered further on). Then, from equation (2),

$$
\mathbf{R}_{y}(\tau)=\boldsymbol{\Phi} \mathbf{R}_{\eta}(\tau) \boldsymbol{\Phi}^{H}+\sigma_{n}^{2} \delta(\tau) \mathbf{I},
$$

where temporally and spatially white noise has been assumed. Since the sources are assumed mutually independent, $\mathbf{R}_{\eta}(\tau)$ should be a diagonal matrix for any value of $\tau$. Therefore, $\boldsymbol{\Phi}$ happens to be the generalised matrix of eigenvectors which diagonalises all matrices $\mathbf{R}_{y}(\tau)$ in a given set of time-lags. This provides the mode shapes of interest. In turn, the diagonal elements of $\mathbf{R}_{\eta}(\tau)$ return the autocorrelation functions of individual modal coordinates, from which global modal parameters can be extracted from standard single-degree-of-freedom techniques.

The physical significance of SOBI when applied to structural responses was investigated in references $[3,4,9,20]$. In particular, it has been pointed out that the assumption of mutually independent (and therefore uncorrelated) modes does not hold true except for conservative systems, i.e. with no damping. However, SOBI happens to be very robust against this assumption, for instance much more than JADE which was historically the first choice to solve the OMA problem [3]. Rather than trying to justify a posteriori the applicability of BSS algorithms to structural dynamics, this paper proposes to follow the other way round. A general approach is proposed which results in the deduction of several new separation criteria based on first physical principles.

\section{Separation from ad hoc criteria - general principles}

This section briefly describes the proposed methodology on a general basis. The effort is put on a deductive presentation, so that the same mechanism can be applied to the various separation criteria to be presented hereafter. First of all, the concept of modal filter is reviewed, since this is where BSS and OMA meet each other. Second, the concept of "action" is introduced for blindly estimating modal filters. Third, a deflation approach to separation is adopted since this has some algorithmic advantages, even though a batch approach may be envisioned as well.

\subsection{The concept of modal filter}

The natural way to inverse equation (1) - or its matrix version $(2)-$ is to find a set of coefficients $w_{i j}$, $i=1, \ldots, n, j=1, \ldots, m$, such that

$$
\hat{\eta}_{i}(t)=\sum_{j=1}^{m} w_{i j}^{*} y_{j}(t)=\mathbf{w}_{i}^{H} \mathbf{y}(t)
$$

(wherein * denotes the conjugate symbol) returns a "good" estimate of the modal coordinate $\eta_{i}(t)$. Vector $\mathbf{w}_{i}$ is referred to as a "spatial" or "modal filter", a concept which largely precedes the introduction of BSS in structural dynamics [26]. These are the primary unknowns of the problem. It now remains to specify how these can be found blindly, i.e. without measuring anything else than the structural responses $y_{i}(t)$ and independently of any parametric model.

\subsection{Action integral}

Inspired by the variational principles of mechanics, let us define a functional $\mathcal{S}(x, \dot{x})$, the "action", which takes an arbitrary signal $x(t)$ and its derivative $\dot{x}(t)$ as inputs. The central idea of the proposed methodology consists in designing $\mathcal{S}(x, \dot{x})$ such that it achieves a minimum if signal $x(t)$ fulfils some physical property of a modal coordinate, that is when $x(t) \equiv \eta_{i}(t)$. After substituting the estimated modal coordinate $\mathbf{w}_{i}^{H} \mathbf{y}(t)$ for its theoretical value $\eta_{i}(t)$, the issue of blindly estimating the modal filter $\mathbf{w}_{i}$ amounts to minimising the following cost function

$$
J(\mathbf{w} ; \mathbf{y})=\mathcal{S}\left(\mathbf{w}^{H} \mathbf{y}, \mathbf{w}^{H} \dot{\mathbf{y}}\right)
$$

with respect to $\mathbf{w}$. In many cases, the above minimisation will be efficiently carried out by means of a gradient descent algorithm (see Appendix). 


\subsection{Iterative separation}

Let us assume that a first modal filter, $\mathbf{w}_{1}$, has been found which minimises $J(\mathbf{w} ; \mathbf{y})$ and therefore provides an estimate $\hat{\eta}_{1}(t)$ of $\eta_{1}(t)$. In order to proceed to the extraction of a second modal coordinate, the effect of the first mode must be subtracted from the structural responses before trying to minimise $J(\mathbf{w} ; \mathbf{y})$ again. This may be achieved as follows. Let $\hat{\boldsymbol{\varphi}}_{1} \hat{\eta}_{1}(t)=\hat{\boldsymbol{\varphi}}_{1} \mathbf{w}_{1}^{H} \mathbf{y}(t)$ be an estimate of the modal responses $\varphi_{1} \eta_{1}(t)$, where mode shape $\boldsymbol{\varphi}_{1}$ stands for the first column of the modal matrix $\boldsymbol{\Phi}$. The minimum mean-square estimate of $\boldsymbol{\varphi}_{1}$ is found as $\hat{\varphi}_{1}=$ $\operatorname{Argmin}_{\boldsymbol{\varphi}}\left\langle\left|\mathbf{y}(t)-\varphi \hat{\eta}_{1}(t)\right|^{2}\right\rangle=\left\langle\mathbf{y}(t) \hat{\eta}_{1}(t)^{*}\right\rangle /\left\langle\left|\hat{\eta}_{1}(t)\right|^{2}\right\rangle$. Therefore,

$$
\mathbf{y}_{.1}(t)=\mathbf{y}(t)-\hat{\boldsymbol{\varphi}}_{1} \hat{\eta}_{1}(t)=\left(\mathbf{I}-\hat{\boldsymbol{\varphi}}_{1} \mathbf{w}_{1}^{H}\right) \mathbf{y}(t)
$$

is an estimate of the structural response free of the effect of mode 1. By repeating the same procedure recursively, one arrives at the following separation algorithm:

step 1:
$\mathbf{w}_{1}=\operatorname{Argmin}_{\mathbf{w}} J(\mathbf{w} ; \mathbf{y})$
$\mathbf{y}_{.0}(t)=\mathbf{y}(t)$
step $k:$
$\hat{\eta}_{k}(t)=\mathbf{w}_{k}^{H} \mathbf{y}_{.(k-1) !}(t)$
$\hat{\boldsymbol{\varphi}}_{k}=\left\langle\mathbf{y}_{.(k-1) !}(t) \hat{\eta}_{k}(t)^{*}\right\rangle /\left\langle\left|\hat{\eta}_{k}(t)\right|^{2}\right\rangle$
$\mathbf{y}_{. k !}(t)=\mathbf{y}_{.(k-1) !}(t)-\hat{\boldsymbol{\varphi}}_{k} \hat{\eta}_{k}(t)=\prod_{i=1}^{k}\left(\mathbf{I}-\hat{\boldsymbol{\varphi}}_{i} \mathbf{w}_{i}^{H}\right) \mathbf{y}(t)$
$\mathbf{w}_{k+1}=\operatorname{Argmin}_{\mathbf{w}} J\left(\mathbf{w} ; \mathbf{y}_{. k !}\right)$
stop when $k=n$.

where $\mathbf{y}_{. k}$ ! $(t)$ denotes an estimate of the structural response free of the effect of modes 1 to $k$. The algorithm directly returns an estimate of the modal matrix, $\hat{\boldsymbol{\Phi}}$, which contains mode shapes $\hat{\boldsymbol{\varphi}}_{k}$ in its $k$-th column, and of the corresponding modal coordinates $\hat{\eta}_{k}(t)$. If needed, an estimate of the overall separation matrix (i.e. the estimated inverse $\hat{\boldsymbol{\Phi}}^{-1}$ of $\boldsymbol{\Phi}$ such that $\left.\hat{\eta}(t)=\hat{\boldsymbol{\Phi}}^{-1} \mathbf{y}(t)\right)$ is returned by the matrix whose $k$ th raw is

$$
\hat{\boldsymbol{\varphi}}^{i}=\mathbf{w}_{k}^{H} \prod_{i=1}^{k}\left(\mathbf{I}-\hat{\boldsymbol{\varphi}}_{i} \mathbf{w}_{i}^{H}\right)
$$

Please note that the series of recursions given by equations (7) to (11) extracts modes one by one without forcing them to be mutually orthogonal (i.e. uncorrelated at time time-lag $\tau=0$ ) contrary to other BSS algorithms such as JADE or SOBI. This removes a strong and artificial condition actually conflicting with the true physics of the problem.

The next section now proposes three least action principles which will furnish relevant candidates for the action $\mathcal{S}(x, \dot{x})$ and its corresponding cost function $J(\mathbf{w} ; \mathbf{y})$.

\section{Least action principles}

A general attribute of a structural mode is to concentrate most of its energy around a specific frequency. This will be declined in three different ways. The first one is inspired from the concept of geodesic in the timedomain, the second one from that of barycentre in the frequency domain, and the third on from that of entropy in the frequency domain. All derivations are made under the assumption of steady state regime and stationary random excitations, yet without restriction (extensions to other scenario, e.g. transient responses, are also possible following similar lines).

Details of the results reported in this section are given in the Appendix.

\subsection{Principle of shortest envelope (SHE)}

Let us construe the modal coordinate $\eta(t)$ as the "trajectory" of the corresponding mode in the time-domain. Let us then define the quadratic envelope of the mode as $\left|\eta_{a}(t)\right|^{2}$, where $\eta_{a}(t)$ denotes the analytical signal version of $\eta(t)$ [27] (see Appendix C.1 on how to compute the analytical signal). The principle of shortest envelope conjectures that, among all possible trajectories, a mode will try to minimise that trajectory with the shortest envelope. More specifically, let $\mathrm{d} l$ be the element of length of the envelope $\left|\eta_{a}(t)\right|^{2}$ corresponding to time increment $\mathrm{d} t$ such that, from the Pythagorean theorem, $\mathrm{d} l^{2}=\mathrm{d} t^{2}+\left(\mathrm{d}\left|\eta_{a}(t)\right|^{2}\right)^{2}$ (see Fig. 1). Thus the total length over a time interval of duration $T$ reads $\int_{T} \mathrm{~d} l=\int_{T} \mathrm{~d} t \sqrt{1+\left(\mathrm{d}\left|\eta_{a}(t)\right|^{2} / \mathrm{d} t\right)^{2}}$. For stationary signals, the action is defined as the average envelope length per unit of time,

$$
\begin{aligned}
\mathcal{S}\left(\eta_{a}, \dot{\eta}_{a}\right) & =\lim _{T \rightarrow \infty} \frac{1}{T} \int_{T} \mathrm{~d} t \sqrt{1+\left(\frac{\mathrm{d}}{\mathrm{d} t}\left|\eta_{a}(t)\right|^{2}\right)^{2}} \\
& =\left\langle\mathcal{L}\left(\eta_{a}, \eta_{a}^{*}, \dot{\eta}_{a}, \dot{\eta}_{a}^{*}\right)\right\rangle \geqslant 1
\end{aligned}
$$

with Lagrangian

$$
\mathcal{L}\left(\eta_{a}, \eta_{a}^{*}, \dot{\eta}_{a}, \dot{\eta}_{a}^{*}\right)=\sqrt{1+4 \Re\left(\dot{\eta}_{a} \eta_{a}^{*}\right)^{2}}
$$

This is schematically illustrated in Figure 1. Note that the quadratic envelope $\left|\eta_{a}(t)\right|^{2}$ was actually considered in equation (13) because it physically reflects fluctuation of energy, a quantity which is naturally minimised 


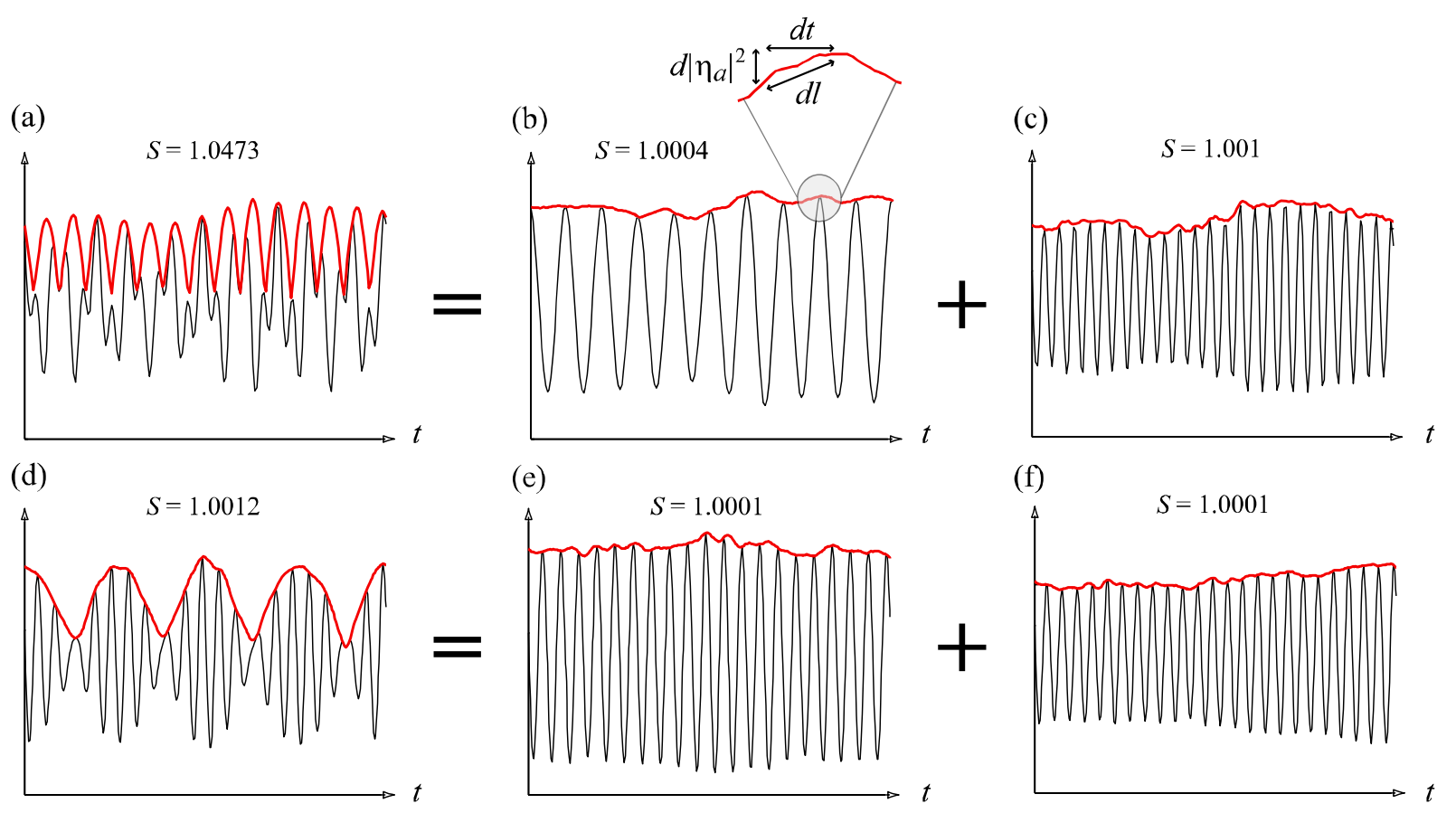

Fig. 1. Illustration of the principle of shortest envelope. Signal (a) is a mixture of two modes with natural frequencies 0.05 and 0.12 (arbitrary units); the length of its envelope is $S=1.0473$. The length of the envelopes of modal constituents shown in (b) and (c) is $S=1.0004$ and $S=1.0010$, respectively. Signals (d-f) bear the same interpretation in the case of two closely space natural frequencies, at 0.05 and 0.06 , which better emphasises the increase in length of the mixture (d) due to a beating phenomenon. Note the theoretical length of a pure sine is $S=1$.

at the thermodynamic equilibrium of a system, and also because it is easily tractable. The envelope magnitude $\left|\eta_{a}(t)\right|$ could have been considered instead of its square, leading to

$$
\begin{aligned}
\mathcal{S}\left(\eta_{a}, \dot{\eta}_{a}\right) & =\lim _{T \rightarrow \infty} \frac{1}{T} \int_{T} \mathrm{~d} t \sqrt{1+\left(\frac{d}{d t}\left|\eta_{a}(t)\right|\right)^{2}} \\
& =\left\langle\mathcal{L}\left(\eta_{a}, \eta_{a}^{*}, \dot{\eta}_{a}, \dot{\eta}_{a}^{*}\right)\right\rangle \geqslant 1
\end{aligned}
$$

with Lagrangian

$$
\mathcal{L}\left(\eta_{a}, \eta_{a}^{*}, \dot{\eta}_{a}, \dot{\eta}_{a}^{*}\right)=\sqrt{1+\frac{\Re\left(\dot{\eta}_{a} \eta_{a}^{*}\right)^{2}}{\left|\eta_{a}\right|^{2}}}
$$

a criterion very similar to equation (14) up to a normalisation by $\left|\eta_{a}\right|^{2}$ under the square root. The authors did not find significant difference between criteria (14) and (16) in terms of separation capability; nevertheless, since it is less involved algorithmically than the latter, the former criterion only will be considered from now on.

Because a mode has no defined magnitude and to avoid the trivial solution, the minimisation of the action must be achieved under the constraint of constant - say unitary - energy $\left\langle\left|\eta_{a}(t)\right|^{2}\right\rangle=1$. Expressed in terms of the unknown modal filter $\mathbf{w}$, action (13) provides the cost function

$$
J\left(\mathbf{w} ; \mathbf{y}_{a}\right)=\left\langle\sqrt{1+4\left(\frac{\Re\left(\mathbf{w}^{H} \dot{\mathbf{y}}_{a}(t) \mathbf{y}_{a}(t)^{H} \mathbf{w}\right)}{\mathbf{w}^{H} \mathbf{R}_{y_{a}} \mathbf{w}}\right)^{2}}\right\rangle
$$

where $\mathbf{y}_{a}$ stands for the analytic signal version of $\mathbf{y}$ and $\mathbf{R}_{y_{a}}=\left\langle\mathbf{y}_{a}(t) \mathbf{y}_{a}(t)^{H}\right\rangle$.

In practice, $J\left(\mathbf{w} ; \mathbf{y}_{a}\right)$ will be easily minimised by means of a descent gradient algorithm, as shown in Appendix C.3. Yet, it is also interesting to work out the minimum from a theoretical point of view by applying the Euler-Lagrange equations to equation (13). After some calculus, this leads to the following "equation of motion"

$$
\frac{\mathrm{d}}{\mathrm{d} t} \Re\left(\dot{\eta}_{a} \eta_{a}^{*}\right)=0
$$

of the mode, to which $\eta_{a}(t)=\mathrm{e}^{i \lambda(t)}, \lambda(t) \in \mathbb{R}$ is, one solution for arbitrary real time-varying phase $\lambda(t)$. In words, pure sinusoids (with possible frequency modulations) are those trajectories with shortest envelope, $\mathcal{S}\left(\eta_{a}, \dot{\eta}_{a}\right)=1$; in terms of geodesics, this corresponds to straight lines. Although actual modal coordinates will never equal pure sinusoids due to the presence of dissipation, this result tells is that the minimisation of $J\left(\mathbf{w} ; \mathbf{y}_{a}\right)$ will separate modes which are the closest possible to sinusoids, which is consistent with the property of modes to be highly concentrated around specific frequencies.

In a more abstract way equation (18) may be seen as the conservation law of quantity $\Re\left(\dot{\eta}_{a} \eta_{a}^{*}\right)$. From Noether's theorem [28], this implies invariance of the Lagrangian (14) under translation and scaling (i.e. change of units) transformations, as expected from the stationarity assumption inherent to the steady state regime and from the normalisation of modal coordinates to unity. 
(a)

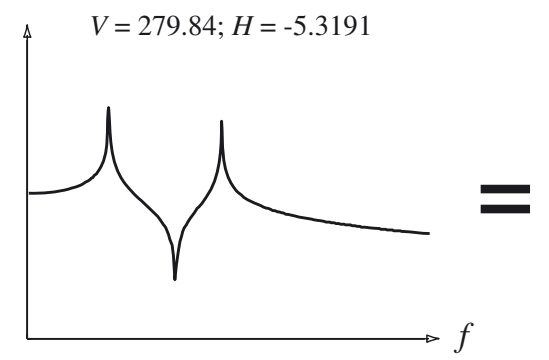

(b)

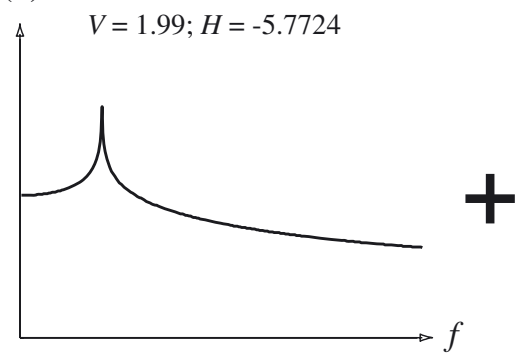

(c)

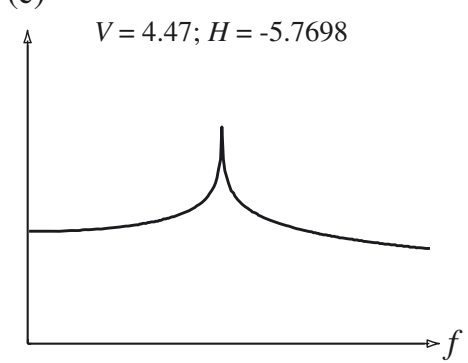

Fig. 2. Illustration of the principles of minimum variance and minimum entropy. Spectrum (a) is a mixture of two modes with natural frequencies 0.05 and 0.12 (arbitrary units); its spectral variance is $V=279.84$ and spectral entropy $H=-5.3191$. The spectral variances and entropies of the modal constituents shown in (b) and (c) are $V=1.99, H=-5.7724$ and $V=4.47$, $H=-5.7698$, respectively.

\subsection{Principle of minimum spectral variance (MSV)}

The principle of shortest envelope proceeds from a time-domain vision of modal responses. A frequency domain vision may be well-suited as well, especially to reflect the property of high energy concentration of a mode around a central frequency. To see this, let us define $S_{\eta}(f)$ the power spectrum (energy spectrum in the case of transient responses) of the modal coordinate $\eta(t)$. Viewing the latter as a probability density function of the mode "trajectory" in frequency, a measure of concentration around the barycentric frequency $f_{0}$ is given by the spectral variance

$$
\frac{\int_{0}^{\infty} S_{\eta}(f)\left(f-f_{0}\right)^{2} \mathrm{~d} f}{\int_{0}^{\infty} S_{\eta}(f) \mathrm{d} f} \geqslant 0 \quad \text { with } \quad f_{0}=\frac{\int_{0}^{\infty} S_{\eta}(f) f \mathrm{~d} f}{\int_{0}^{\infty} S_{\eta}(f) \mathrm{d} f}
$$

This is illustrated in Figure 2. Converted back to the timedomain by use of the Parseval's identity, this returns the action (see Appendix A)

$$
\mathcal{S}\left(\eta_{a}, \dot{\eta}_{a}\right)=\left\langle\left|\eta_{a}\right|^{2}\right\rangle\left\langle\left|\dot{\eta}_{a}\right|^{2}\right\rangle-\left\langle\Im\left(\dot{\eta}_{a} \eta_{a}^{*}\right)\right\rangle^{2}
$$

Thus, the modal filter w should achieve the minimum of the following cost function

$$
J\left(\mathbf{w} ; \mathbf{y}_{a}\right)=\frac{\mathbf{w}^{H} \mathbf{R}_{\dot{y}_{a}} \mathbf{w}}{\mathbf{w}^{H} \mathbf{R}_{y_{a}} \mathbf{w}}-\left(\frac{\Im\left(\mathbf{w}^{H} \mathbf{R}_{\dot{y}_{a} y_{a}} \mathbf{w}\right)}{\mathbf{w}^{H} \mathbf{R}_{y_{a}} \mathbf{w}}\right)^{2}
$$

where $\quad \mathbf{R}_{\dot{y}_{a}}=\left\langle\dot{\mathbf{y}}_{a}(t) \dot{\mathbf{y}}_{a}(t)^{H}\right\rangle$ and $\mathbf{R}_{\dot{y}_{a} y_{a}}=$ $\left\langle\dot{\mathbf{y}}_{a}(t) \mathbf{y}_{a}(t)^{H}\right\rangle$.

In contrast to equation (13), action (20) is difficult to express in terms of a Lagrangian. However it does not prevent application of calculus of variation from producing the "equation of motion" (see Appendix B)

$$
-\ddot{\eta}_{a}\left\langle\left|\eta_{a}\right|^{2}\right\rangle+2 i \dot{\eta}_{a}\left\langle\Im\left(\dot{\eta}_{a} \eta_{a}^{*}\right)\right\rangle+\eta_{a}\left\langle\left|\dot{\eta}_{a}\right|^{2}\right\rangle=0
$$

of the mode, to which $\eta_{a}(t)=\mathrm{e}^{i(\lambda t+\varphi)}, \lambda \in \mathbb{R}$, is found as a solution with $\mathcal{S}\left(\eta_{a}, \dot{\eta}_{a}\right)=0$. Note this is more restrictive than the solution previously found for the principle of shortest envelope since it precludes frequency modulation, yet at the same time it is also more physical in this respect. Indeed, for this particular solution, the action

$$
\mathcal{S}\left(\eta_{a}, \dot{\eta}_{a}\right)=\left\langle\left|\dot{\eta}_{a}\right|^{2}\right\rangle+\lambda^{2} \underbrace{\left\langle\left|\eta_{a}\right|^{2}\right\rangle}_{=1}=2(K+U)
$$

boils down to (twice) the total energy of a simple oscillator with mass 1 , kinetic energy $K=\frac{1}{2}\left\langle\left|\dot{\eta}_{a}\right|^{2}\right\rangle$, potential energy $U=\frac{1}{2} \lambda^{2}\left\langle\left|\eta_{a}\right|^{2}\right\rangle$, and stiffness $\lambda^{2}$. Therefore minimising the spectral variance amounts to minimising the kinetic energy $K$ of the mode given a constant potential energy $U=\frac{1}{2} \lambda^{2}$ (remember the constraint $\left\langle\left|\eta_{a}\right|^{2}\right\rangle=1$ ).

\subsection{Principle of minimum spectral entropy (MSEnt)}

Keeping on with the interpretation of the power spectrum $S_{\eta}(f)$ as the probability density function of the trajectory of a mode in the frequency-domain (see Fig. 2), another least action principle is to conjecture that the trajectory should be the less erratic as possible, that is with the least entropy

$$
H\left(S_{\eta}\right)=-\int_{0}^{\infty} \frac{S_{\eta}(f)}{\int_{0}^{\infty} S_{\eta}(f) \mathrm{d} f} \ln \left(\frac{S_{\eta}(f)}{\int_{0}^{\infty} S_{\eta}(f) \mathrm{d} f}\right) \mathrm{d} f .
$$

The motivation of this conjecture is as follows. As known from the field of information theory, the largest possible entropy will correspond to a flat spectrum, $S_{\eta}(f)=\sigma_{\eta}^{2}$, whereas the smallest possible one will correspond to a delta spectrum, $S_{\eta}(f)=A^{2} \delta\left(f-f_{0}\right)$, i.e. a pure line. The principle of minimum spectral entropy will therefore again favour highly concentrated structures in frequency - that is pure sinusoids in the time-domain. Equation (24) also shares a strong similarity with equation (19), where in both cases the minimization is achieved by squeezing the mode resonance by suitably tuning a notch filter: in the former case the notch filter has the parabolic shape $\left(f-f_{0}\right)^{2}$, whereas in the former it is directly returned by the "inverted resonance" $-\ln S_{\eta}(f)$.

The time domain counterpart of $H\left(S_{\eta}\right)$, the action $\mathcal{S}\left(\eta_{a}, \dot{\eta}_{a}\right)$, is unfortunately difficult to express and does 
not actually give further insight. For this reason, this third least action principle will not be assigned an equation of motion. The corresponding cost function

$$
J\left(\mathbf{w} ; \mathbf{y}_{a}\right)=-\int_{0}^{\infty} \frac{\mathbf{w}^{H} \mathbf{S}_{y}(f) \mathbf{w}}{\mathbf{w}^{H} \mathbf{R}_{y_{a}} \mathbf{w}} \ln \left(\frac{\mathbf{w}^{H} \mathbf{S}_{y}(f) \mathbf{w}}{\mathbf{w}^{H} \mathbf{R}_{y_{a}} \mathbf{w}}\right) \mathrm{d} f
$$

will directly be minimised with respect to $\mathbf{w}$, where $\mathbf{S}_{y}(f)$ denotes the spectral matrix of the measurements and where the Parseval's identify $\int_{0}^{\infty} \mathbf{S}_{y}(f) d f=\mathbf{R}_{y_{a}}$ has been used.

\subsection{Discussion}

Several remarks are in order at this juncture. First, the fact that the proposed least action criteria do not depend on the structural parameters (e.g. mass, stiffness and damping values) cannot be overemphasised. Indeed, this complies with the BSS philosophy to be able to separate the independent components passing through a system without any knowledge of the system itself. It also explains why the homogeneous "equations of motion" (18) and (22) are quite different from the usual differential equation, $\ddot{\eta}_{a}+2 \omega_{0} \zeta_{0} \dot{\eta}_{a}+\omega_{0}^{2} \eta_{a}=f$, which requires knowledge of parameters $\omega_{0}$ and $\zeta_{0}$ and of the unknown forcing function $f(t)$.

Second, it was found that minima of criteria (13), (19) and (24) are returned by pure sines which, strictly speaking, are solutions of conservative systems only. Fortunately it does not mean that the proposed criteria will fail in the presence of energy dissipation, but simply that they will try to separate the most lightly damped modes among all possible solutions. Again this perfectly complies with the physical definition of a mode, to which criteria (19) and (24) are quite explicit in this respect (they minimise the modal bandwidth which is inversely proportional to the damping ratio). Intuitively, a lightly damped mode will produce slowly modulated sinusoids that are quite close to the theoretical solutions. Specifically, let

$$
\eta_{a}(t)=a(t) \mathrm{e}^{j\left(\omega_{0} t+\phi(t)\right)}
$$

be the polar form of the modal response $\eta_{a}(t)$, where $a(t)$ stands for amplitude modulation and $\phi(t)$ for phase modulations around the natural frequency $\omega_{0}$ of the mode. Note that the amount of damping will directly affect the rapidity of the fluctuations in $a(t)$ and $\phi(t)$ : the lighter the damping, the slower the modulations. Then, it is readily checked that the principles of shortest envelope and of minimum spectral variance minimise the quantities

$$
\left\langle\sqrt{1+\frac{\dot{a} a}{\left\langle a^{2}\right\rangle}}\right\rangle
$$

and

$$
\left\langle\frac{\left\langle\dot{a}^{2}\right\rangle}{\left\langle a^{2}\right\rangle}+\left\langle\left(\omega_{0}+\dot{\phi}\right)^{2}\right\rangle\right\rangle
$$

respectively. This means the later tries to separate modes with least amplitude and phase fluctuations, whereas the later bothers about amplitude fluctuation only. In other words, components are separated which are as lightly damped as possible, i.e. as close as possible to sinusoids. This proves to some extent the validity of the proposed criteria in the presence of dissipation. However, from a purely numerical point of view, it is clear that the degree of damping will directly impact upon the convergence rate when minimising the proposed criteria. This will be demonstrated in Section 5.

Third, although the case of steady state regime has been assumed (stationary signals) hitherto, the proposed principles are not at all limited to that specific configuration and apply just as well to transient responses with minor modification in the corresponding algorithms. This issue is not carried on here.

Finally, it is noteworthy that the principle of least envelope happens to be more general than the two other ones, for it also accepts modal contributions with modulated frequency as solutions. This opens interesting perspectives in all application concerned with time-varying or non-linear systems $[27,29]$, which are not investigated here due to lack of space.

\section{Experimental validations}

The three proposed principles are now demonstrated and validated on both synthetic and real data. A particular attention is paid to comparisons with SOBI which stands as a point of reference in the literature; as a consequence, this section is not intended to bring any comparison with experimental modal analysis (i.e. when excitations are measured) since this has already been covered in several other research works.

\subsection{Parametric analyses}

This subsection aims at comparing, through simulations, the principles of shortest envelope, minimum spectral variance, and minimum spectral entropy with SOBI, a BSS algorithm introduced in Section 2 which serves as a point of reference in OMA.

The data are synthesised by first numerically simulating the steady state acceleration responses $\eta_{i}(t), i=$ $1, \ldots, n$ of $n$ uncoupled oscillators to independent and identically distributed (white Gaussian noise) excitations, then mixing them with a $n \times n$ random (Gaussian) modal matrix $\boldsymbol{\Phi}$, and finally adding additive white Gaussian noise $\mathbf{n}(t)$ to produce the system responses given by equation (2).

The separation algorithms are then run with a tentative number of degrees of freedom, $n_{e}$, which may differ from the actual one, $n$. Care is taken to assign the same stopping rule to each algorithm (e.g. a fixed threshold on the relative error norm of the estimated separation matrix). In all experiments SOBI is first run with 2 times-lags 
(a)

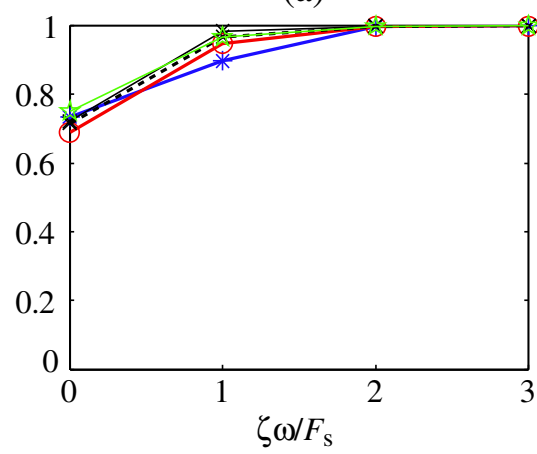

(b)

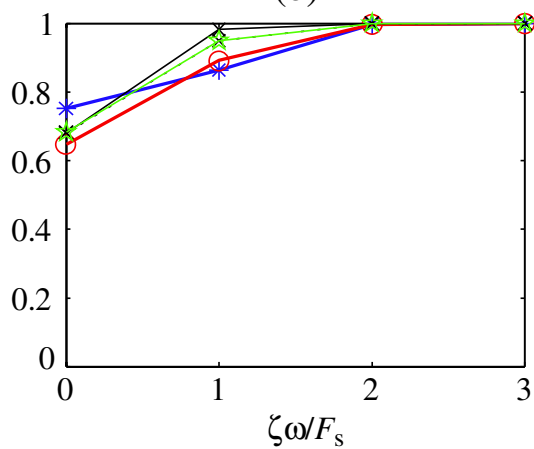

(c)

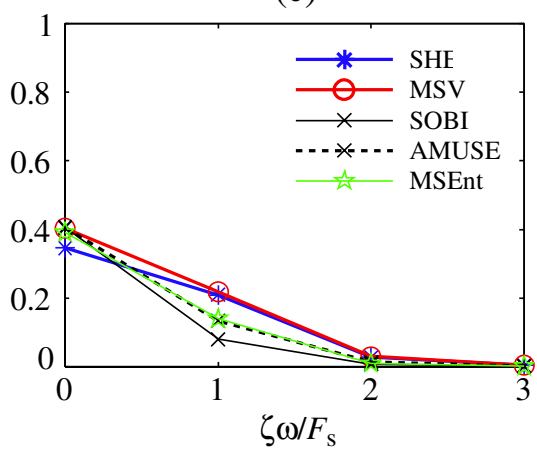

Fig. 3. Comparison of separation results for increasing values of damping $\zeta \omega / F_{s}$ with $\mathrm{SNR}=20 \mathrm{~dB}$ and $m=n=n_{e}=4$ : (a) correlation coefficient, (b) MAC, (c) relative "cross-talk" error.

(a)

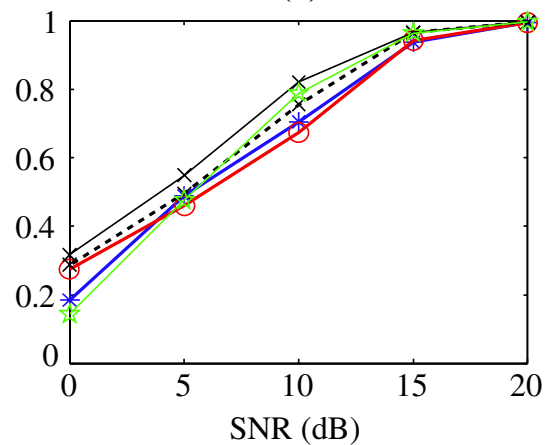

(b)

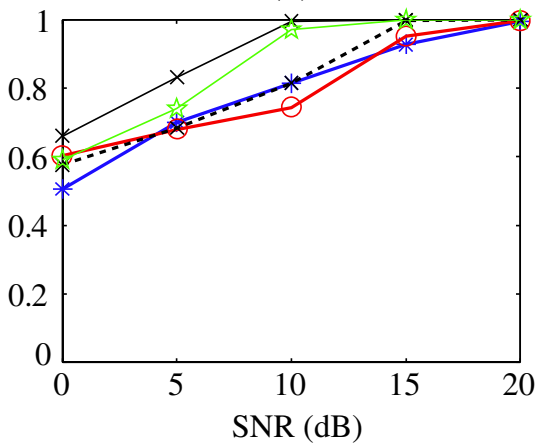

(c)

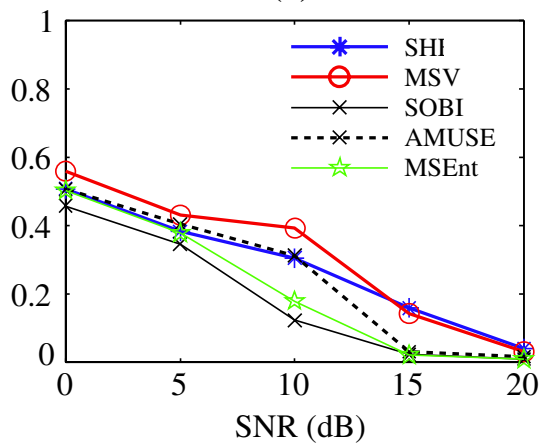

Fig. 4. Comparison of separation results for increasing values of SNR with $\zeta \omega / F_{s}=10^{-2}$ and $m=n=n_{e}=4$ : (a) correlation coefficient, (b) MAC, (c) relative "cross-talk" error.

$\tau=\{0,1\}$ (which corresponds to the so-called AMUSE algorithm [25]) and next with 10 times-lags $\tau=\{0, \ldots, 9\}$. The following figures of merit are displayed:

(a) the correlation coefficient, $0 \leqslant \rho^{2} \leqslant 1$, between the separated modal coordinates and the actual ones;

(b) the MAC (Modal Acceptance Criterion) between the estimated mode shapes (columns of separation matrix) and the actual ones;

(c) the relative energy of "cross-talk" errors

$$
e=\sqrt{\frac{1}{n(n-1)} \sum_{i=1}^{n} \sum_{j \neq i}\left|\frac{\hat{\boldsymbol{\varphi}}^{i} \boldsymbol{\varphi}_{j}}{\hat{\boldsymbol{\varphi}}^{i} \boldsymbol{\varphi}_{i}}\right|^{2}}
$$

where $\hat{\boldsymbol{\varphi}}^{i}$ is the $i$ th raw of the separation matrix (12), which is ideally zero for perfect separation.

Please note all these statistics are invariant under mode scaling, a necessary condition to cope with the fundamental indeterminacy of BSS. In each experiment the figures of merit are averaged over 100 realisations of different modal matrices, oscillator excitations, and additive noise.

The first experiment takes $m=n=n_{e}=4$ and compares the results for increasing values $\zeta \omega F_{s}\left(F_{s}=\right.$ sampling frequency) of the damping in the system (see Fig. 3). The signal-to-noise-ratio (SNR) is $20 \mathrm{~dB}$ and the natural frequencies of the modal coordinates are $0.1 F_{s}$,
$0.1587 F_{s}, 0.2520 F_{s}$, and $0.4 F_{s}$. The second experiment takes a fixed damping $\zeta \omega / F_{s}=10^{-2}$ and compares the results for increasing values of the SNR for the same system settings as before (see Fig. 4). The third experiment compares the results for increasing values of the condition number ${ }^{1} \kappa$ of the modal matrix (see Fig. 5 ). Finally, the fourth experiment compares the results for increasing values of the number $n$ of degrees of freedom of the systems $\left(m=n=n_{e}\right)$ given $n$ natural frequencies logarithmically spaced from $0.1 F_{s}$ to $0.4 F_{s}$ (see Fig. 6).

All experiments show that the proposed least action criteria have behaviour very similar to SOBI with 2 timelags: namely, separation results are all the better as damping is light [20], the SNR is high, and the condition number is low. The fact that separation tends to be more difficult in the presence of many degrees of freedom is probably to be blamed more on algorithmic issues than on the criteria themselves and, in that respect, the matrix implementation of SOBI seems slightly inferior to the deflation approach proposed here. Incidentally, the deflation approach will always separate the same first $k$ modes whatever the estimated total number of active modes, $n_{e} \geqslant k$, which is not the case of SOBI where the latter has an influence on the separation results. Considering

1 The condition number of the modal matrix was imposed by artificially scaling the logarithm of its singular values. 
(a)

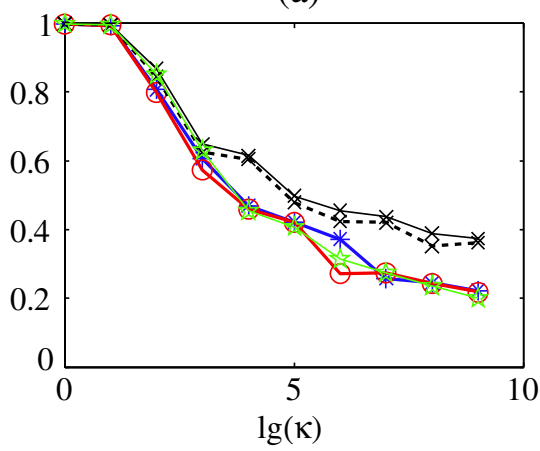

(b)

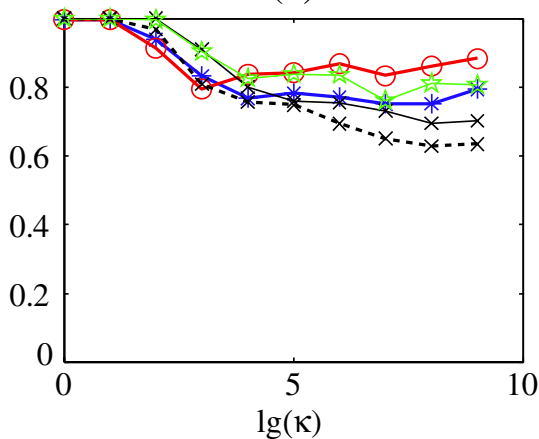

(c)

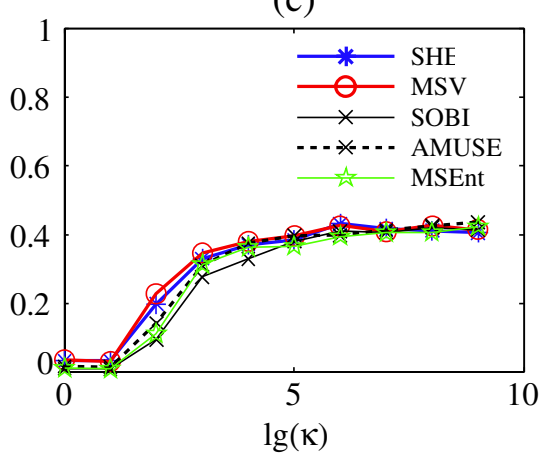

Fig. 5. Comparison of separation results for increasing condition $\kappa$ numbers with $\zeta \omega / F_{s}=10^{-2}, \mathrm{SNR}=20 \mathrm{~dB}$, and $m=n=$ $n_{e}=4$ : (a) correlation coefficient, (b) MAC, (c) relative "cross-talk" error.

(a)

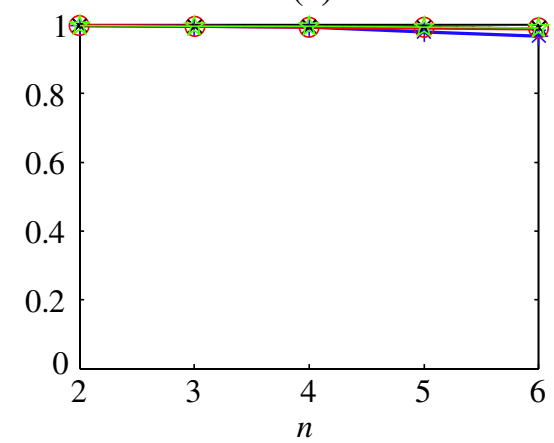

(b)

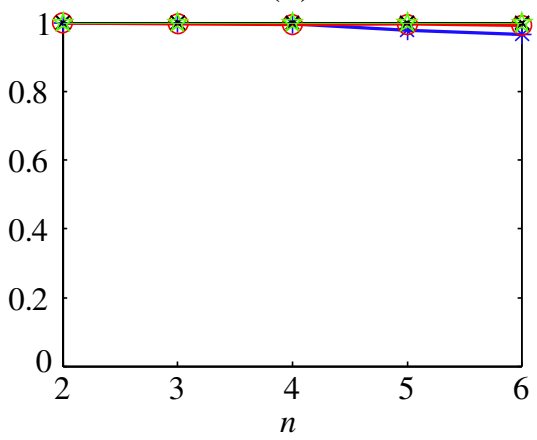

(c)

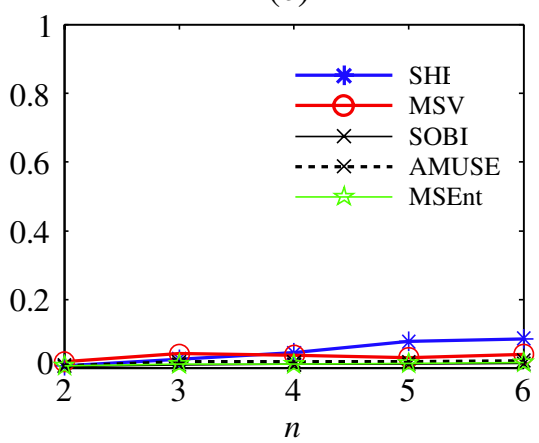

Fig. 6. Comparison of separation results for increasing numbers of degrees of freedom $n$ with $\zeta \omega / F_{s}=10^{-2}$, SNR $=20 \mathrm{~dB}$, and $m=n=n_{e}$ : (a) correlation coefficient, (b) MAC, (c) relative "cross-talk" error.

the difficulty of setting a priori (and somewhat arbitrarily) the value of $n_{e}$ in practice - in particular because $n_{e}$ will typically underestimate the actual value of actives modes, $n$ - this appears a definite advantage over SOBI and other similar batch algorithms. Next, it is noteworthy that SOBI with 10 time-lags evidences a slightly better performance than other criteria in all situations, which is consistent with the fact that, in some sense, it combines 9 criteria instead of one. Finally, among the proposed criteria, the principle of minimum spectral entropy seems to display the best performances, even though it is based on the same physical principle as the principle of minimum spectral variance. This is probably due to the use of the logarithm in its definition which can better handle large dynamics between modes than a linear description. However, experiments carried out in the next sections will not confirm the generality of this superiority.

\subsection{Example of application to synthetic data}

This subsection demonstrates the application of the proposed criteria on a simulated 15 degree-of-freedom system purposely designed to produce complex, heavily damped, and strongly coupled modes - a configuration which was demonstrated to be unfavourable to SOBI in reference [20] - as well as some local modes. The system and its corresponding mode shapes - are displayed in Figure 7 .

Mass, stiffness and damping values are $m_{i}=$ $10 / 386.09 \mathrm{~kg}, i=1, \ldots, 10, m_{i}=0.5 / 386.09 \mathrm{~kg}, i=11$, $\ldots, 15, k=1000 \mathrm{~N} / \mathrm{m}, c_{1}=c_{2}=0.20 \mathrm{~kg} / \mathrm{s}, c_{3}=$ $0.05 \mathrm{~kg} / \mathrm{s}$, respectively. The system is excited by white Gaussian forces and acceleration responses are collected at all $m=15$ degrees of freedom. Signals are sampled at $T_{s}^{-1}=1024 \mathrm{~Hz}$ for a total of 163840 samples. Figure 8 displays the power spectral densities (weighted with a 512-long Hanning window) of the system responses and of the modal coordinates separated by the proposed least action criteria. Separation with SOBI is not shown because it is qualitatively very similar to Figure 8b (see Ref. [20] for a thorough investigation of SOBI on the same example). It is seen that all criteria return satisfactory separation of the 15 modal coordinates, despite some difficulties for the principle of minimum spectral entropy to resolve between strongly couples modes. Figures of merit of the separation algorithms are returned in Table 1. Surprisingly, the principle of minimum spectral entropy is seen to reach the lowest cross-talk error among the proposed least action criteria although the separated spectra were not perfect. This is actually not contradictory, since the separated spectra reflect the estimation of the modal coordinates whereas the cross-talk error is indicative of the estimation of the mode shapes; thus very different 

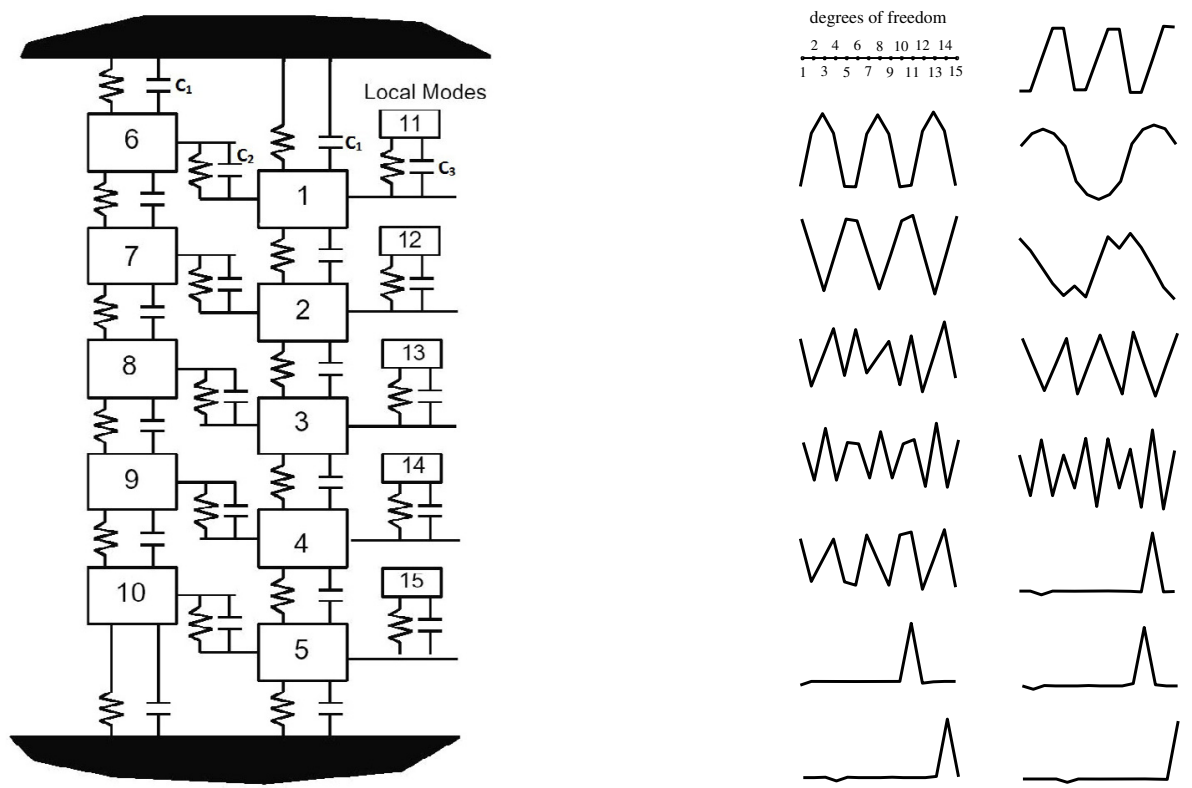

Fig. 7. (a) Simulated 15 degree-of-freedom system and (b) corresponding mode shapes.

(a)

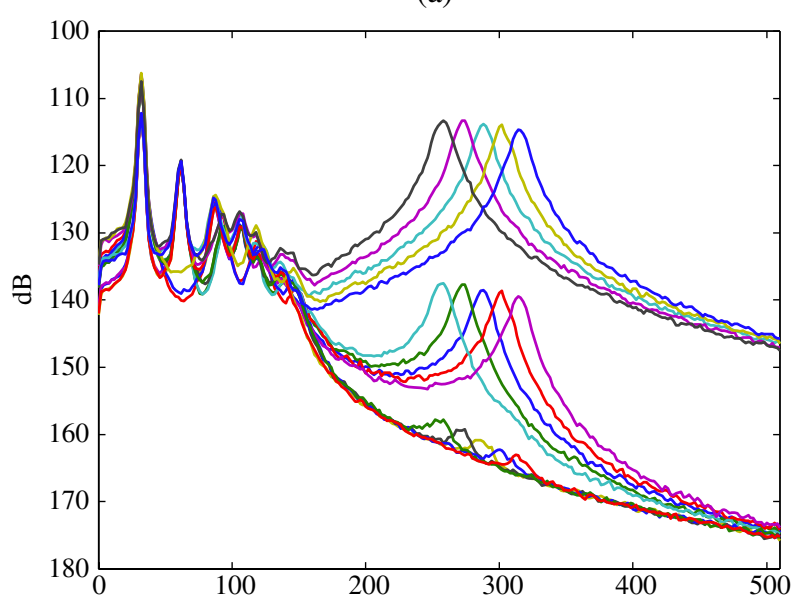

(c)

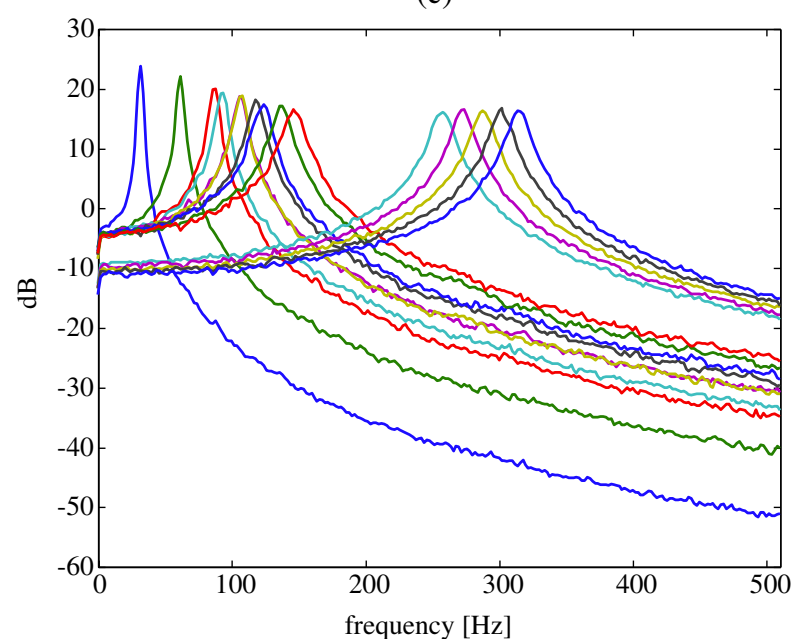

(b)

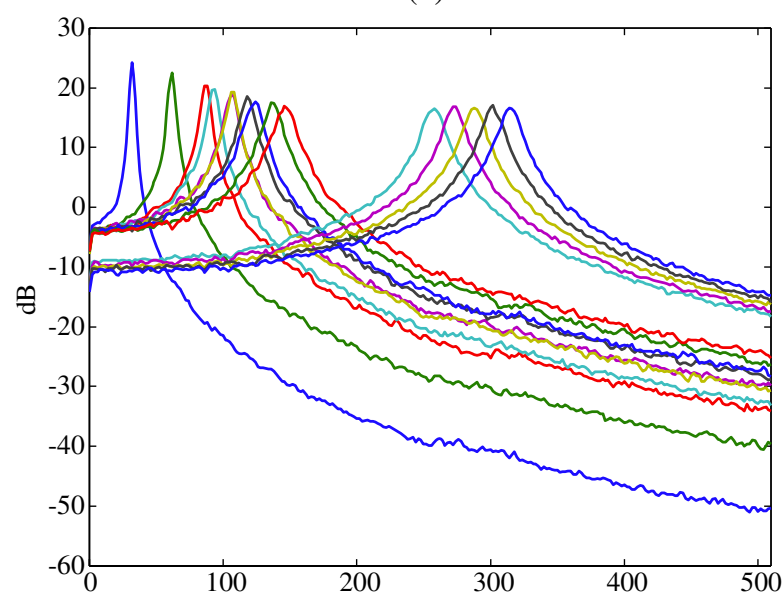

(d)

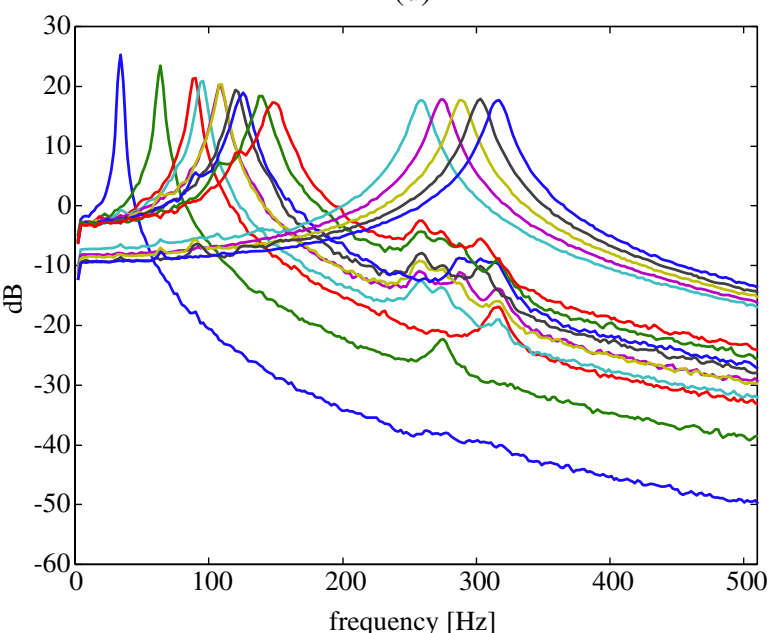

Fig. 8. Power spectra of (a) the system responses and of the separated modal coordinates by (b) SHE, (c) MSV, and (d) MSEnt (frequency resolution $\Delta f=2 \mathrm{~Hz}$ ). 
Table 1. Figures of merit of the separation algorithms.

\begin{tabular}{cccccc}
\hline $\begin{array}{c}\text { Separation } \\
\text { method }\end{array}$ & $\begin{array}{c}\text { SOBI (with 10 time- } \\
\text { lags) }\end{array}$ & $\begin{array}{c}\text { SOBI (with 2 time- } \\
\text { lags) }\end{array}$ & SHE & MSV & MSEnt \\
\hline Cross-talk error & 0.0369 & 0.0389 & 0.0597 & 0.0565 & 0.0526 \\
\hline
\end{tabular}

SOBI

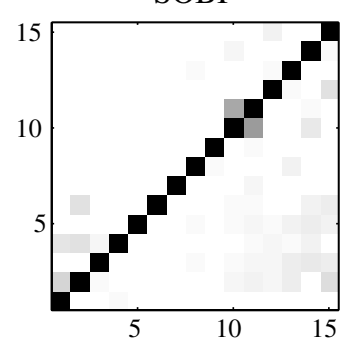

MSV

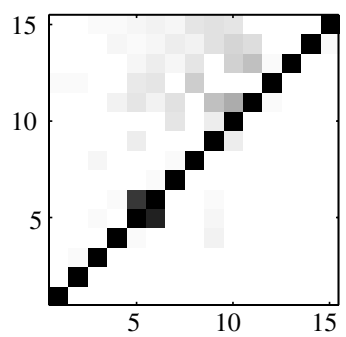

SHE
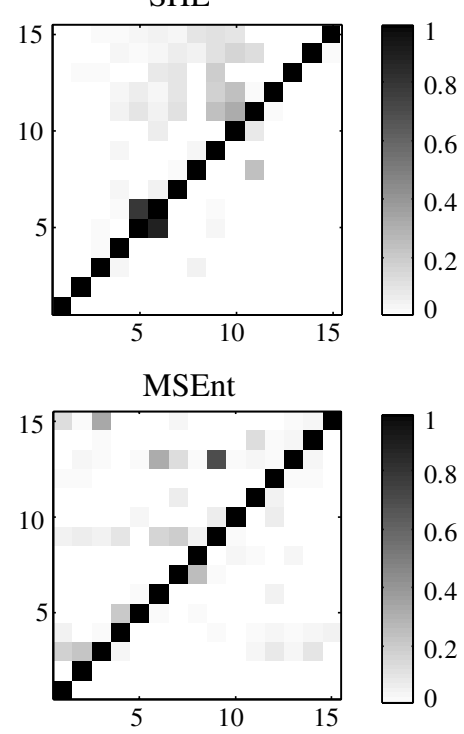

Fig. 9. Matrices of values $e_{i j}=\left|\hat{\varphi}^{i} \boldsymbol{\varphi}_{j}\right| /\left|\hat{\varphi}^{i} \boldsymbol{\varphi}_{i}\right|$ reflecting the closeness of the separation matrix to the actual inverse of the modal matrix $\left(e_{i j}\right.$ are ideally the elements of the identify matrix).

quantities are considered. Note that these figures of merit are actually subjected to small random fluctuations due to random initialisation of the algorithm of Section 3.3 and therefore are statistically very similar. However, the cross-talk errors of the least action criteria are, on the average, about 1.5 as large as that of SOBI (whether used with 2 or 10 time-lags) in this example. Finally, Figure 9 displays the individual quantities

$$
e_{i j}=\left|\frac{\hat{\boldsymbol{\varphi}}^{i} \boldsymbol{\varphi}_{j}}{\hat{\boldsymbol{\varphi}}^{i} \boldsymbol{\varphi}_{i}}\right|
$$

which reflect the closeness of the separation matrix (12) to the actual inverse $\boldsymbol{\Phi}^{-1}$ of the modal matrix $\left(e_{i j}\right.$ should be ideally one for $i=j$ and zero otherwise).

\subsection{Example of application to real data}

This last subsection illustrates the least action criteria on a set of data provided for modal parameter estimation round robin on occasion of the IMAC XXVII 2011 conference [30]. A scaled model of a wind turbine blade (see Fig. 10) was considered to evaluate the performance of OMA techniques on a simple experimental structure. The structure was fixed at the root and excited by means of random tapping. Responses were measured in all three directions at 16 locations, with sampling rate of $512 \mathrm{~Hz}$,

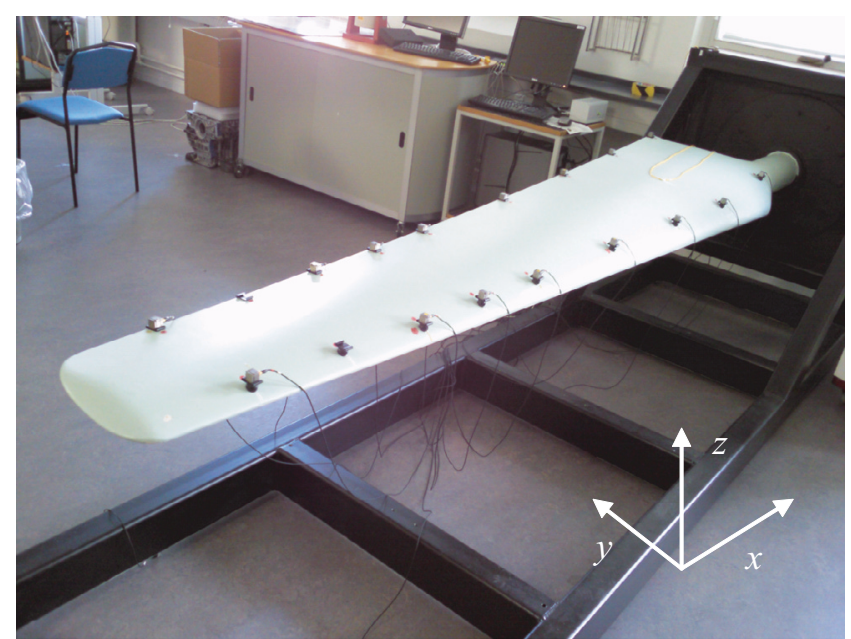

Fig. 10. Scaled model of wind turbine blade.

for a total of 156160 samples per channel. Figure 11 displays the power spectral densities (weighted with a 512long Hanning window) of the system responses and of the modal coordinates separated by SOBI (with 10 timelags) and by the proposed least action criteria. On the one hand, very satisfactory separation of 10 modal coordinates is achieved, with natural frequencies of about $13 \mathrm{~Hz}, 68 \mathrm{~Hz}, 112 \mathrm{~Hz}, 124 \mathrm{~Hz}, 149 \mathrm{~Hz}, 160 \mathrm{~Hz}, 181 \mathrm{~Hz}$, $193 \mathrm{~Hz}, 216 \mathrm{~Hz}, 234 \mathrm{~Hz}$; on the other hand, 6 separated signals (not shown in Fig. 11) could easily be recognised as "numerical modes" because of non-physical features and thus were removed. Comparison of the criteria is difficult without point of reference and, indeed, seems to depend on the frequency band of interest. Finally, Figure 12 displays the estimated mode shapes returned by the principle of minimum spectral variance (very similar results were obtained from other criteria).

\section{Conclusion}

BSS was lately recognised as a potential solution to OMA. This discovery may be qualified as "accidental", since algorithms initially developed in and for the field of (telecommunication) signal processing were then transposed and tested with some success to structural dynamics. This led to a number of attempts to justify a posteriori their physical relevance. The goal of this paper is to show that the route could have been taken the other way round and separation algorithms deduced from first physical principles. Three principles of least action have been proposed in this endeavour: the principles of shortest envelope, of minimum spectral variance, and of minimum spectral entropy. The algorithmic implementation 
(a)

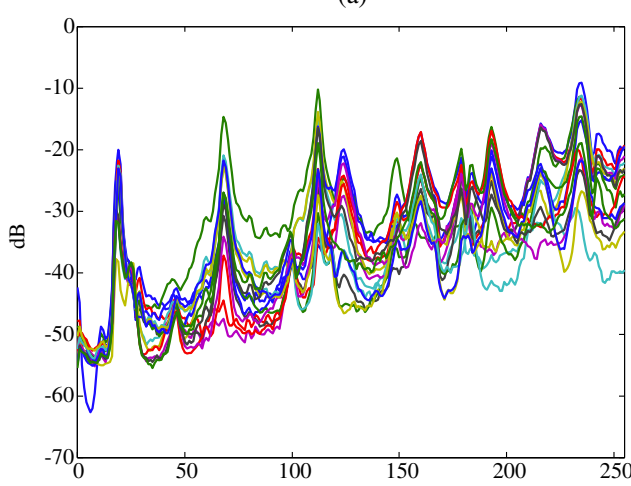

(c)

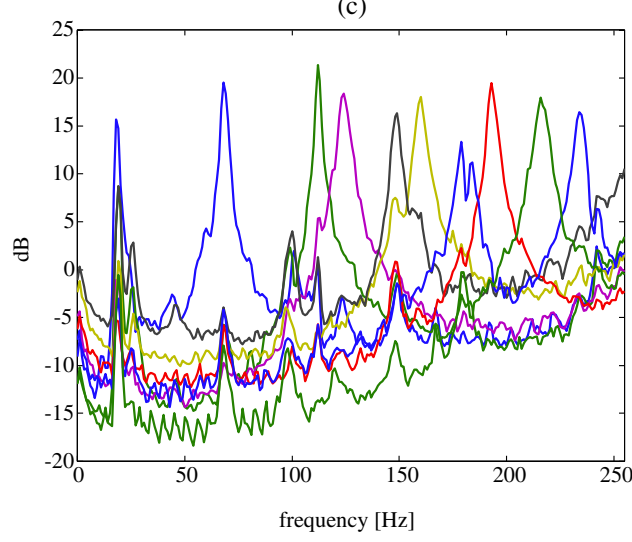

(b)

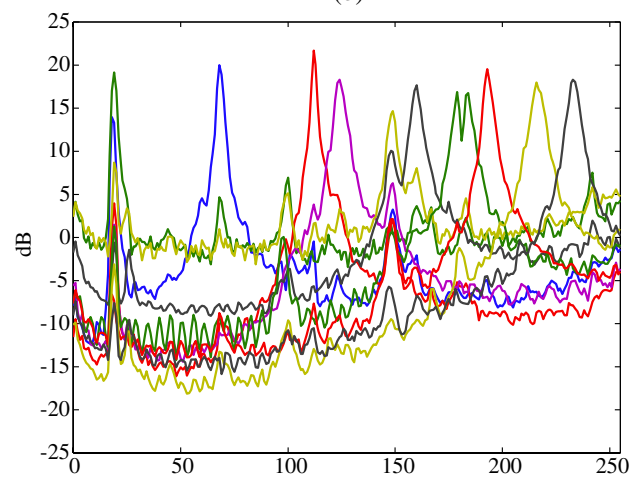

(d)

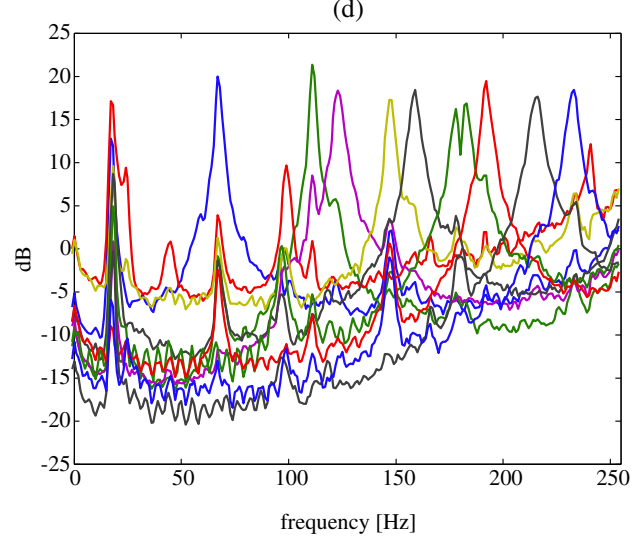

Fig. 11. Power spectra of (a) the system responses and of the separated modal coordinates by (b) SHE, (c) MSV, and (d) MSEnt (frequency resolution $\Delta f=1 \mathrm{~Hz}$ ).
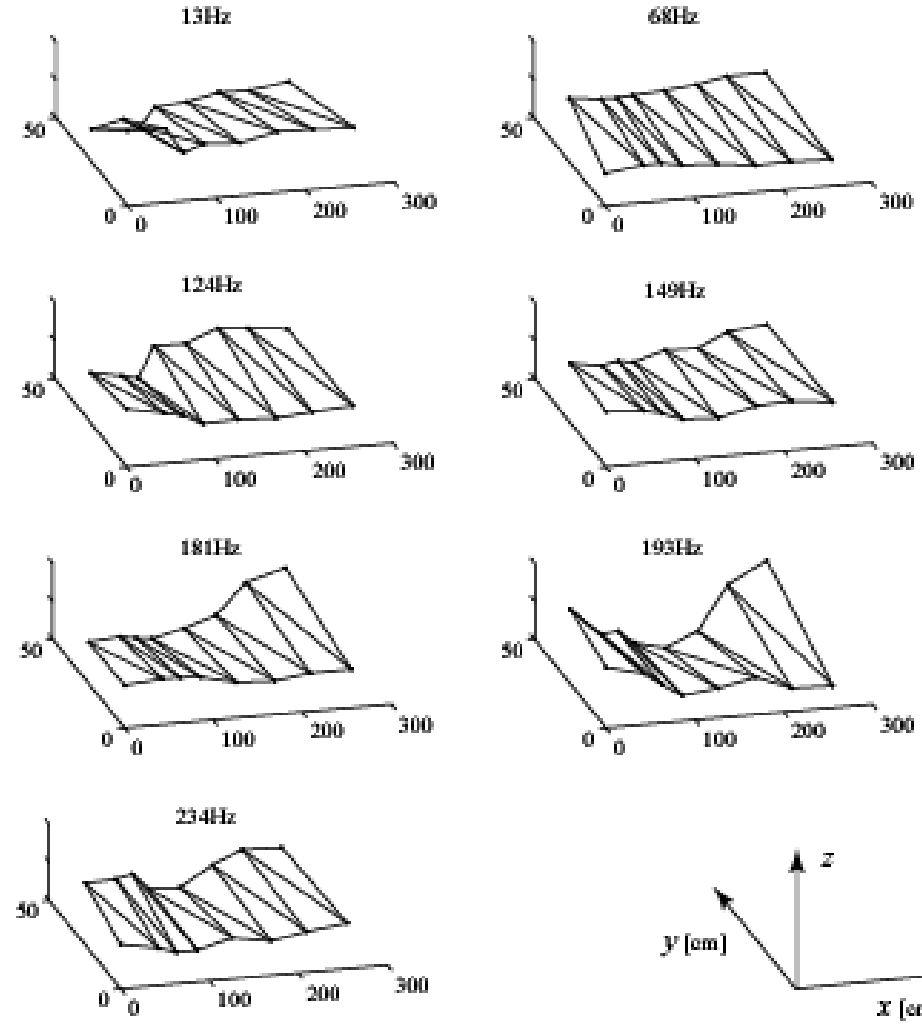
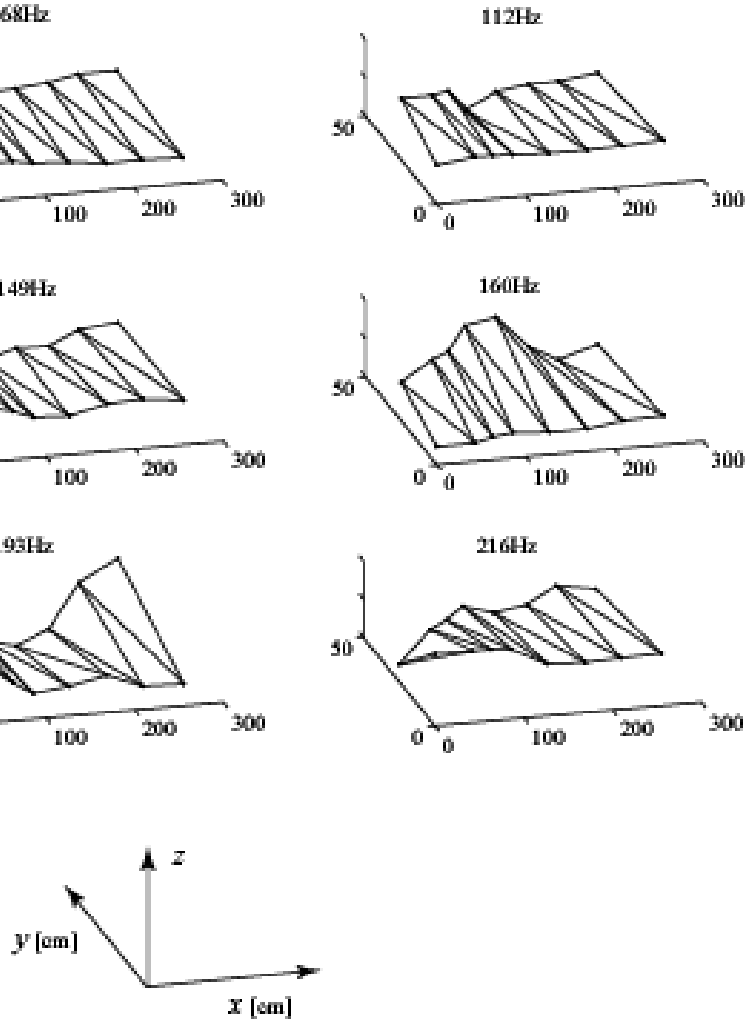

Fig. 12. Estimated mode shapes from MSV. 
of these criteria naturally leads to a deflation approach where modes are extracted one by one, a definite advantage when their actual number is not known a priori. Extensive simulations have shown that the proposed criteria lead to figures of merit very similar to those of SOBI, a point of reference in the current state-of-the-art. Their main advantage is yet conceptual rather than algorithmic; indeed, least action principles provide physical insight into the mechanism of BSS that other algorithms issued form the signal processing community may dramatically lack. No doubt that other physical properties could be exploited to define still new separation criteria by following the lines of the paper. Another perspective is to combine the proposed criteria into a single one (or to apply a criterion on the successive derivatives of the same signal which should preserve unchanged the properties of a mode) in order to improve the overall robustness. As a final remark, it is reminded that the results of the paper have been established under the steady-state regime (stationary signals), yet extension to transient regimes should be no problem.

\section{Appendix A: Proof of equation (20)}

Equation (19) is first rewritten as:

$$
\frac{\int_{-\infty}^{+\infty} S_{\eta_{a}}(f) f^{2} \mathrm{~d} f-\left(\int_{-\infty}^{+\infty} S_{\eta_{a}}(f) f \mathrm{~d} f\right)^{2}}{\int_{-\infty}^{+\infty} S_{\eta_{a}}(f) \mathrm{d} f}
$$

where the integration is now from $-\infty$ to $+\infty$ thanks to the substitution of the analytical signal $\eta_{a}(t)$ in place of the real signal $\eta(t)$. Now, because $(j 2 \pi f)$ is the frequency response function of the time derivative operator, it comes that $S_{\dot{\eta}_{a}}(f)=4 \pi^{2} S_{\eta_{a}}(f) f^{2}$ and $S_{\dot{\eta}_{a} \eta_{a}}(f)=j 2 \pi S_{\eta_{a}}(f) f$ where $S_{\dot{\eta}_{a}}(f)$ stands for the power spectrum of $\dot{\eta}_{a}(t)=$ $d \eta_{a}(t) / d t$ and $S_{\dot{\eta}_{a} \eta_{a}}(f)$ for the cross-spectrum of $\dot{\eta}_{a}(t)$ and $\eta_{a}(t)$. Therefore, equation (A.1) becomes

$$
\frac{\int_{-\infty}^{+\infty} S_{\dot{\eta}_{a}}(f) \mathrm{d} f-\Im\left(\int_{-\infty}^{+\infty} S_{\dot{\eta}_{a} \eta_{a}}(f) \mathrm{d} f\right)^{2}}{4 \pi^{2} \int_{-\infty}^{+\infty} S_{\eta_{a}}(f) \mathrm{d} f}
$$

Finally, using Parseval's identify, $\int_{-\infty}^{+\infty} S_{\eta_{a}}(f) \mathrm{d} f=$ $\left\langle\left|\eta_{a}\right|^{2}\right\rangle, \int_{-\infty}^{+\infty} S_{\dot{\eta}_{a}}(f) \mathrm{d} f=\left\langle\left|\dot{\eta}_{a}\right|^{2}\right\rangle$, and $\int_{-\infty}^{+\infty} S_{\dot{\eta}_{a} \eta_{a}}(f) \mathrm{d} f=$ $\left\langle\dot{\eta}_{a} \eta_{a}^{*}\right\rangle$. Since the action is defined up to a constant, multiplying by $4 \pi^{2}\left\langle\left|\eta_{a}\right|^{2}\right\rangle$ then returns equation (20).

\section{Appendix B: Calculus of variation}

This appendix introduces calculus of variation for stationary and complex-valued signals. If the action integral is defined as the time average

$$
\begin{aligned}
\mathcal{S}(x, \dot{x}) & =\lim _{T \rightarrow \infty} \frac{1}{T} \int_{t_{0}}^{t_{0}+T} \mathrm{~d} t \mathcal{L}\left(x, x^{*}, \dot{x}, \dot{x}^{*} ; t\right) \\
& =\left\langle\mathcal{L}\left(x, x^{*}, \dot{x}, \dot{x}^{*} ; t\right)\right\rangle
\end{aligned}
$$

of a real Lagrangian $\mathcal{L}\left(x, x^{*}, \dot{x}, \dot{x}^{*} ; t\right)$, then EulerLagrange's equation reads

$$
\frac{\partial}{\partial x} \mathcal{L}=\frac{\mathrm{d}}{\mathrm{d} t} \frac{\partial}{\partial \dot{x}} \mathcal{L}
$$

independently of signal $x$ being complex. The initial conditions $x\left(t_{0}\right)$ and $\dot{x}\left(t_{0}\right)$ can be set arbitrary since

$\lim _{T \rightarrow \infty} \frac{1}{T}\left(\left.\frac{\partial \mathcal{L}}{\partial \dot{x}}\right|_{t_{0}} \delta x\left(t_{0}\right)\right)=\left(\left.\frac{\partial \mathcal{L}}{\partial \dot{x}}\right|_{t_{0}} \delta x\left(t_{0}\right)\right) \times \lim _{T \rightarrow \infty} \frac{1}{T}=0$

and similarly at $t_{0}+T$. The equation of motion (18) immediately follows by application of these results to equation (14). Arriving at equation (20) needs a slightly different route since action (20) is not associated with a Lagrangian in the form of equation (B.1). The stationary point of the action is then sought by setting

$$
\delta \mathcal{S}(x, \dot{x})=\mathcal{S}\left(x+\delta x^{*}, \dot{x}+\delta \dot{x}^{*}\right)-\mathcal{S}(x, \dot{x})=0
$$

where $\delta x^{*}$ and $\delta \dot{x}^{*}$ are infinitesimal perturbations on the conjugate signal $x^{*}$. Application of the above condition to action (20) yields

$$
\begin{aligned}
\left\langle\dot{\eta}_{a} \delta \dot{\eta}_{a}^{*}\right\rangle\left\langle\left|\eta_{a}\right|^{2}\right\rangle & +\left\langle\eta_{a} \delta \eta_{a}^{*}\right\rangle\left\langle\left|\dot{\eta}_{a}\right|^{2}\right\rangle \\
& +\mathrm{i}\left\langle\Im\left(\dot{\eta}_{a} \eta_{a}^{*}\right)\right\rangle\left\langle\dot{\eta}_{a} \delta \eta_{a}^{*}-\eta_{a} \delta \dot{\eta}_{a}^{*}\right\rangle=0 .
\end{aligned}
$$

Using integration by parts together with the same argument that led to equation (B.3), one finds $\left\langle\dot{\eta}_{a} \delta \dot{\eta}_{a}^{*}\right\rangle=$ $-\left\langle\ddot{\eta}_{a} \delta \eta_{a}^{*}\right\rangle$. Therefore

$$
\begin{aligned}
& -\left\langle\ddot{\eta}_{a} \delta \eta_{a}^{*}\right\rangle\left\langle\left|\eta_{a}\right|^{2}\right\rangle+\left\langle\eta_{a} \delta \eta_{a}^{*}\right\rangle\left\langle\left|\dot{\eta}_{a}\right|^{2}\right\rangle \\
& +\mathrm{i}\left\langle\Im\left(\dot{\eta}_{a} \eta_{a}^{*}\right)\right\rangle\left\langle\dot{\eta}_{a} \delta \eta_{a}^{*}+\dot{\eta}_{a} \delta \eta_{a}^{*}\right\rangle=0
\end{aligned}
$$

The fact that this result must hold whatever the value of $\delta x^{*}$ finally proves equation (22).

\section{Appendix C: Methodological guidelines}

The aim of this appendix is to provide some methodological guidelines as how to implement the minimisation of the proposed criteria.

\section{C.1. Analytical signal}

All criteria have been devised with complex modal coordinate $\eta_{a}(t)$ of which the complex exponential $\mathrm{e}^{j \lambda t}$ is a particular solution. Physically speaking, this constrains the space of solutions to have positive frequencies only. This is not only convenient in the formulation of the criteria, but it also allows the recovery of complex mode shapes to some extent, as demonstrated in reference [7], although this is not carried on in the present paper. From an algorithmic point of view, the constraint is easily forced by taking the analytical versions of the structural responses $y_{i}(t), i=1, \ldots, m$, that is by zeroing the negative frequencies in their Fourier transforms. 


\section{C.2. Discrete time}

Although the proposed criteria have been devised in the continuous time setting, they will apply to discretetime signals in practice. On the one hand, this is no problem at all for the frequency domain criterion (25) where the spectral matrix $\mathbf{S}_{y}(f)$ is simply to be computed from the discrete Fourier transform. On the other hand, because they operate in the time-domain, criteria (17) and (21) need a specific discretisation scheme of the derivative operator $\mathrm{d} / \mathrm{d} t$. Let $y[n]$ denote the discrete version of $y(t)$ at time $t=n T_{s}$ with $T_{s}$ the sampling period. Simulations have shown that a reasonable approximation of the time derivative $\dot{\mathbf{y}}_{a}(t)$ in criterion (17) is returned by the simple finite difference $\left(\mathbf{y}_{a}[n]-\mathbf{y}_{a}[n-1]\right) / T_{s}$. Criterion (21) requires more care since it has been verified that higher-order schemes are necessary. In the present work, the derivative was approximated by a FIR filter with 21 coefficients,

$$
\dot{\mathbf{y}}_{a}\left(n T_{s}\right) \approx \frac{1}{T_{s}} \sum_{k=-10}^{10} w_{k} \frac{(-1)^{k}}{k} \mathbf{y}_{a}[n-k]
$$

where $w_{k}$ is a Hanning window.

\section{C.3. Gradients}

Criteria (17), (21) and (25) can be efficiently minimised with a gradient descent algorithm. This subsection provides the required gradient expressions assuming the modal filters are real-valued. Let $q_{0}=\mathbf{w}^{H} \mathbf{R}_{y_{a}} \mathbf{w}=$ $\sum_{i, j=1}^{n} w_{i} w_{j} R_{i j}$ and $q_{1}(t)=2 \Re\left(\mathbf{w}^{H} \dot{\mathbf{y}}_{a}(t) \mathbf{y}_{a}(t)^{H} \mathbf{w}\right)=$ $2 \sum_{i, j=1}^{n} w_{i} w_{j} \Re\left(\dot{y}_{a, i}(t) y_{a, j}^{*}(t)\right)$ with $w_{i} \in \Re$. Then, the gradient of criteria (17) with respect to the $i$ th component $w_{i}$ of the modal filter $\mathbf{w}$ reads

$$
\frac{\partial J}{\partial w_{i}}=\left\langle q_{1}(t) \frac{q_{0} \partial_{i} q_{1}(t)-q_{1}(t) \partial_{i} q_{0}}{\sqrt{q_{0}^{2}+q_{1}(t)^{2}}}\right\rangle
$$

with

$$
\partial_{i} q_{0}=\frac{\partial q_{0}}{\partial w_{i}}=2 \sum_{j=1}^{n} w_{j} \Re\left(R_{i j}\right)
$$

and

$$
\partial_{i} q_{1}(t)=\frac{\partial q_{1}(t)}{\partial w_{i}}=4 \sum_{j=1}^{n} w_{j} \Re\left(\dot{y}_{a, i}(t) y_{a, j}^{*}(t)\right)
$$

Let us now define $q_{2}(t)=\Im\left(\mathbf{w}^{H} \mathbf{R}_{\dot{y}_{a} y_{a}} \mathbf{w}\right)=$ $\sum_{i, j=1}^{n} w_{i} w_{j} \Im\left(\dot{y}_{i, a}(t) \dot{y}_{j, a}^{*}(t)\right)$. Then, the gradient of criteria (21) reads

$$
\begin{aligned}
\frac{\partial J}{\partial w_{i}}=\frac{q_{0}\left\langle\partial_{i} q_{1}(t)\right\rangle}{} & -\left\langle q_{1}(t)\right\rangle \partial_{i} q_{0} \\
& q_{0}^{2} \\
& \times\left(q_{0}\left\langle\partial_{i} q_{2}(t)\right\rangle-\left\langle q_{2}(t)\right\rangle\right. \\
q_{0}^{3} & \\
&
\end{aligned}
$$

with

$\partial_{i} q_{2}(t)=\frac{\partial q_{2}(t)}{\partial w_{i}^{*}}=\sum_{j=1}^{n} w_{j} \Im\left(\dot{y}_{i, a}(t) \dot{y}_{j, a}^{*}(t)+\dot{y}_{j, a}(t) \dot{y}_{i, a}^{*}(t)\right)$

Finally, let $Q(f)=\mathbf{w}^{H} \mathbf{S}_{y}(f) \mathbf{w}=\sum_{i, j=1}^{n} w_{i} w_{j} S_{y_{i} y_{j}}(f)$. Then, the gradient of criteria (25) reads

$$
\frac{\partial J}{\partial w_{i}}=\frac{1}{q_{0}^{2}} \int_{0}^{\infty}\left(Q(f) \partial_{i} q_{0}-q_{0} \partial_{i} Q(f)\right)\left(1+\ln \frac{Q(f)}{q_{0}}\right) \mathrm{d} f
$$

with

$$
\partial_{i} Q(f)=\frac{\partial Q(f)}{\partial w_{i}}=2 \sum_{j=1}^{n} w_{j} \Re\left(S_{y_{i} y_{j}}(f)\right) .
$$

\section{References}

[1] P. Van Overschee, B. De Moor, Subspace Identification for Linear Systems: Theory-ImplementationsApplications, Kluwer Academic Publishers, Dordrecht, Netherlands, 1996

[2] R. Brincker, P. Andersen, Understanding stochastic subspace identification, Proceedings of the 24th IMAC, St. Louis, Missouri, 2006

[3] G. Kerschen, F. Poncelet, J.-C. Golinval, Physical interpretation of independent component analysis in structural dynamics, Mech. Syst. Signal Process. 21 (2007) $1561-1575$

[4] F. Poncelet, G. Kerschen, J.-C. Golinval, D. Verhelst, Output-only modal analysis using blind source separation techniques", Mech. Syst. Signal Process. 21 (2007) $2335-2358$

[5] W. Zhou, D. Chelidze, Blind source separation based vibration mode identification, Mech. Syst. Signal Process. 21 (2007) 3072-3087

[6] S. Chauhan, R. Martell, R.J. Allemang, D.L. Brown, Application of independent component analysis and blind source separation techniques to operational modal analysis, Proceedings of the 25th IMAC, Orlando (FL), USA, 2007

[7] S.I. McNiell, D.C. Zimmerman, A Framework for blind modal identification using joint approximate diagonalization, Mech. Syst. Signal Process. 22 (2008) 1526-1548

[8] Hazra, A.J. Roffel, S. Narasimhan, M.D. Pandey, Modified cross-correlation method for the blind identification of structures, J. Eng. Mech. 136 (2010) 889-897

[9] S.I. McNeill, D.C. Zimmerman, Relating independent components to free-vibration modal responses, Shock and Vibration 17 (2010) 161-170

[10] Swaminathan, B. Sharma, S. Chauhan, Utilization of blind source separation techniques for modal analysis, Proceedings of the 28th IMAC, Jacksonville (FL), USA, 2010

[11] H. Jing, H.-Q. Yuan, F.-G. Wu, Research on dynamic response of MDOF systems using independent component analysis, Wuhan Ligong Daxue Xuebao/Journal of Wuhan University of Technology 32 (2010) 68-72 
[12] X.-D. Zhang, Q.-F. Yao, Method of modal parameters identification based on blind sources separation, Zhendong yu Chongji/Journal of Vibration and Shock 29 (2010) 150-153

[13] H. Jing, H.-Q. Yuan, Y. Zhao, Structural modal parameter identification based on independent component analysis, Zhendong yu Chongji/Journal of Vibration and Shock 29 (2010) 137-141

[14] Z.-C. Fu, W. Cheng, C. Xu, Modal parameter identification via robust second-order blind identification method, Zhendong yu Chongji/Journal of Vibration and Shock 29 (2010) 108-111

[15] V.H. Nguyen, J.C. Golinval, Damage detection using blind source separation techniques, Proceedings of the 29th IMAC, Jacksonville (FL), USA, 2011

[16] J. Antoni, S. Braun, Special Issue: Blind Source Separation, Mech. Syst. Signal Process. 19 (2005) $1163-1380$

[17] J. Antoni, Blind separation of vibration components: principles and demonstrations, Mech. Syst. Signal Process. 19 (2005) 1166-1180

[18] J. Antoni, S. Chauhan, Second Order Blind Source Separation techniques (SO-BSS) and their relation to Stochastic Subspace Identification (SSI) algorithm, IMAC XXVIII (International Modal Analysis Conference), Jacksonville, Florida, 2010

[19] J. Antoni, S. Chauhan, An Alternating Least Squares (ALS) based Blind Source Separation Algorithm for Operational Modal Analysis, IMAC XXIX (International Modal Analysis Conference), Jacksonville, Florida, 2011
[20] J. Antoni, S. Chauhan, A study and extension of secondorder blind source separation to operational modal analysis, J. Sound Vib. 332 (2013) 1079-1106

[21] A. Belouchrani, K. Abed-Meraim, J.-F. Cardoso, E. Moulines, A blind source separation technique using second order statistics, IEEE Trans. Signal Process. 45 (1997) pp. 434-444

[22] J.-F. Cardoso, Blind signal separation: statistical principles, Proc. IEEE 86 (1998) 2009-2025

[23] Hyvarinen, E. Oja, Independent component analysis: algorithms and applications, Neural Netw. 13 (2000) $411-430$

[24] Hyvarinen, J. Karhunen, E. Oja, Independent Component Analysis, John Wiley and Sons, New York, 2001

[25] A. Cichocki, S. Amari, Adaptive blind signal and image processing, John Wiley and Sons, New York, 2002

[26] S.J. Shelly, Investigation of discrete modal filters for structural dynamic applications, $\mathrm{PhD}$ Dissertation, Department of Mechanical, Industrial and Nuclear Engineering, University of Cincinnati, 1991

[27] M. Feldman, Hilbert Transform Applications in Mechanical Vibration, Wiley, 2011

[28] V.I. Arnold, Mathematical Methods of Classical Mechanics, Springer, New York, 1989

[29] A. Bellino, A. Fasana, E. Gandino, L. Garibaldi, S. Marchesiello, A time-varying inertia pendulum: modelling and experiments, Mech. Syst. Signal Process., Available online 22 May 2013

[30] http://www.me.mtu.edu/imac_oma/ (last visit 08/08/2013) 